\title{
Fuzzy sentiment analysis using convolutional neural network
}

Cite as: AIP Conference Proceedings 2329, 050002 (2021); https://doi.org/10.1063/5.0042144

Published Online: 26 February 2021

Sugiyarto, Joko Eliyanto, Nursyiva Irsalinda, and Meita Fitrianawati

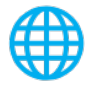

\section{ARTICLES YOU MAY BE INTERESTED IN}

Crowdsourcing as a tool to elicit software requirements

AIP Conference Proceedings 2329, 050001 (2021); https://doi.org/10.1063/5.0042134

Classification of mycobacterium tuberculosis based on color feature extraction using adaptive boosting method

AIP Conference Proceedings 2329, 050005 (2021); https://doi.org/10.1063/5.0042283

Public health on social media: Using Instagram posts for investigating dengue hemorrhagic fever in Indonesia

AIP Conference Proceedings 2329, 050004 (2021); https://doi.org/10.1063/5.0042267

Challenge us.

What are your needs for periodic signal detection?

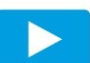

Watch

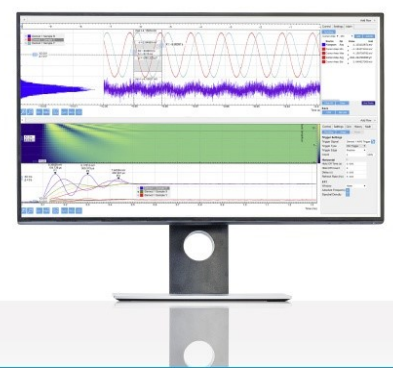

- Zurich - Instruments 


\title{
Fuzzy Sentiment Analysis Using Convolutional Neural Network
}

\author{
Sugiyarto $^{1}$, Joko Eliyanto ${ }^{2, a)}$, Nursyiva Irsalinda $^{1}$ and Meita Fitrianawati ${ }^{3}$ \\ ${ }^{1}$ Mathematics Department, Faculty of Applied Science and Technology, Universitas Ahmad Dahlan, Indonesia \\ ${ }^{2}$ Mathematics Education Department, Postgraduate Program, Universitas Ahmad Dahlan, Indonesia \\ ${ }^{3}$ Elementary School Teacher Study Program, Faculty of Teacher Training and Education, \\ Universitas Ahmad Dahlan, Indonesia \\ ${ }^{a)}$ Corresponding author: joko1907050003@webmail.uad.ac.id
}

\begin{abstract}
Sentiment analysis is one part of natural language processing. Sentiment analysis can be done by lexicon based, or machine learning based. Sentiment analysis based on machine learning has advantage of dynamism to meet with new language datasets or new vocabulary. Sentiment analysis seeks to understand the sentiments contained in a sentence. A sentence can be positive, neutral or negative, based on its sentiments. A sentence can have positive, neutral or negative sentiments. However, the fact is each sentence does not always have positive, negative or neutral sentiment clearly. We try to develop a sentiment analysis method that can show the sentiment degree of a sentence. Fuzzy sentiment analysis using convolutional neural network are introduced in this paper to produce more accurate sentiment analysis results. Convolutional neural networks are a popular machine learning method for sentiment analysis. The concept of fuzzy sets is used to express the sentiment degree of a sentence. Euclidean distance analysis to determine the proximity of two vectors is used to show that this method is better than the standard method. The method we propose successfully produces a value that indicates the degree of sentiment of a sentence. Comparison of the euclid distance between the results of the standard sentiment analysis and our method shows that the results of the fuzzy sentiment analysis using convolutional neural network have a distance that is relatively close to the true sentiment value. Fuzzy convolutional neural network analysis sentiment is proven to be able to produce better and smoother sentiment analysis results than standard methods.
\end{abstract}

\section{INTRODUCTION}

Natural language processing is an area of research and application that explores how computers can be used to understand and manipulate language texts for useful purposes [1]. Sentiment analysis is one part of natural language processing [2]. Sentiment analysis seeks to understand the sentiments contained in a sentence. A sentence can be positive, neutral or negative based on its sentiments [3]. However, the fact is each sentence does not always have positive, negative or neutral sentiment clearly. (However, in fact, each sentence does not always have positive, negative or neutral sentiment clearly.) Sentiment analysis is widely used in the field of marketing to determine consumer reactions and to determine the public response to a policy taken by the government [4]. There are various approaches to conducting sentiment analysis.

Sentiment analysis can be done with lexicon based or machine learning based [5]. Machine learning works based on algorithms on (in) classification data that is labeled manually. This data trained using machine learning is very influential on the resulting classification model, the greater dataset will give the better result. Thus, sentiment analysis based on machine learning has advantage of dynamism to meet with new language datasets or new vocabulary. This approach is better than a lexicon-based approach [5]. One popular algorithm in machine learning is convolutional neural networks. 
Convolutional neural networks is one of the branches of deep learning that has the best level of accuracy based on some research in previous [6]. This method can be applied to carry out sentiment analysis. Y. Zhang and B. Wallace used word2vec for pre-training, area filter size, and several feature maps and get the result in an accuracy of up to $81.65 \%$ for sentiment analysis [7]. A. Hassan and A. Mahmood tried to combine Convolutional Neural Networks with Long Short Term Memoryfor the training process. The combination of the two methods shows an accuracy of up to $88.3 \%$ [8]. With the same goal, research [9] implemented one hot encoding for pre-training. With a predetermined hyperparameter value, the results of the study show the use of smaller filter sizes gives an accuracy of up to $77.4 \%$. The smaller filter size has a positive impact on increasing training accuracy and time [9]. CNN experiments with three layers of convolution and adding word $2 \mathrm{vec}$ pre-training were conducted by [10] with a more complicated architecture it turns out that only produces an accuracy of $45.4 \%$.

However, the result of a sentiment analysis is a clear or hard classification. The classification is negative, positive, and neutral [11]. In the CNN model, the classification results are generated by selecting the highest probability value in the output of each class. Another form of sentiment analysis results is sentiment score, this result is a positive, negative or zero integer value. The results of sentiment analysis are based on these numbers [12]. However, this sentiment score is generated in the dictionary-based sentiment analysis. In fact, the sentiment value in a sentence is not entirely positive, negative or neutral. There is always ambiguity there. The concept of fuzzy logic is a good concept to illustrate this point.

The fuzzy logic model is based on the fuzzy set theory proposed by Zadeh [13]. In the standard logic model, the truth value is expressly stated by the numbers 0 and 1 , but in this model, the truth value of a sentence is the value in the interval [0,1] [14]. The application of fuzzy logic has been tried to improve machine learning algorithms for sentiment analysis $[15,16]$. In other studies, fuzzy logic is applied to the initial domain in the fuzzy convolutional neural networks method [17]. Sentiment analysis developed based on fuzzy logic has also been investigated [18-23]. This shows that fuzzy logic in sentiment analysis is very relevant to be developed at this time.

In this study, we focus on developing machine learning-based sentiment analysis with convolutional neural networks algorithms that produce sentiment degrees. This algorithm has advantages for feature extraction and produce high accuracy for sentiment analysis. The degree of sentiment will better describe the ambiguity of the sentiment of a word or sentence. The main problem that we want to solve is how to present sentiment analysis in the form of sentiment degrees. Besides that, we also want to produce a dynamic sentiment analysis method for large datasets and high accuracy. Our goal is to produce sentiment values based on fuzzy logic, i.e. (that is,) sentiment values are in the range of 0 and 1 . Words or sentences with negative sentiment are 0 and sentences with positive sentiment are 1. Sentences of neutral sentiment are around 0.5. In addition we also aim to produce analytical sentiments that have high accuracy and dynamism towards the addition of text datasets. This method is applied to a dataset that is adjusted to the desired conditions. Namely a dataset in the form of text that has been labeled based on the value of sentiment. This method was developed to modify the output results on the convolutional neural networks algorithm so that it produces sentiment values in fuzzy form.

\section{METHODS}

This research was conducted on the TensorFlow 2.0 module in the Pyhton version 3 programming language [24]. The text consisting of characters will be prepared and encoded in a series of one-dimensional numbers. This input will be train using the convolutional neural networks algorithm. The results of this training process are models that will produce predictive probabilities for each class (eg positive and negative or positive, negative, and neutral). This output will be modified in fuzzy form so that sentiment values are obtained which represent the sentiment degree of a sentence. Finally, we use euclid distance between the sentiment vector and the sentiment vector prediction to show that our result is approaching sentiment value.

\section{Machine Learning Approach for Sentiment Analysis}

Sentiment analysis with machine learning approach is part of supervised learning [25]. Every sentence or text, labeled by its sentiment. It can be binary (negative and positive) or multi-class (negative, neutral, and positive). Generally the text is standardized by cleaning process. The unnecessary characters are removed. Then tokenization is applied. Tokenization is the process of breaking a sentence into words. Then the words are encoded. Each word is represented by a single number, look at Fig. 1(a). These sentences, will be formed into rows of numbers in a vector. Before going to the next process, these vectors are uniform in length. This process is called padding, look at Fig. 
1(b). The final result of this preparation process is a matrix of texts and sentiment vectors. Sentiment values are converted into category vectors. This final form will be broken up into two parts, namely training and test dataset with the desired comparison.

Tokenization

Input:

One of the other reviewers

$$
\begin{aligned}
& \text { Output: } \\
& \left\{\begin{aligned}
\{1 & \text { : 'one', } \\
2 & \text { : 'of', } \\
3 & \text { : 'the', } \\
4 & \text { : 'other', } \\
5 & \text { : 'reviewers'\} }
\end{aligned}\right.
\end{aligned}
$$

(a)

\section{Encoding + Padding}

Input:

I love this movie

\section{Output:}

\#Encoding

$[17,30,76,15]$

\#Encoding+padding

$[17,30,76,15,0,0, \ldots, 0]$

(b)

FIGURE 1. Preparation process on sentimen analysis

The machine learning algorithm will train the training data for getting (to get) a pattern. After that, it will bevalidate with test dataset. The final result of this process is a sentiment model that provide the value of training accuracy and validation accuracy. To improve the quality of the model, (There are) several ways can be applied. First by adding and expanding the type of input data or modify the arrangement of layers in the convolutional neural network model. A diagram of this sentiment analysis using machine learning approach is shown in Fig. 2.

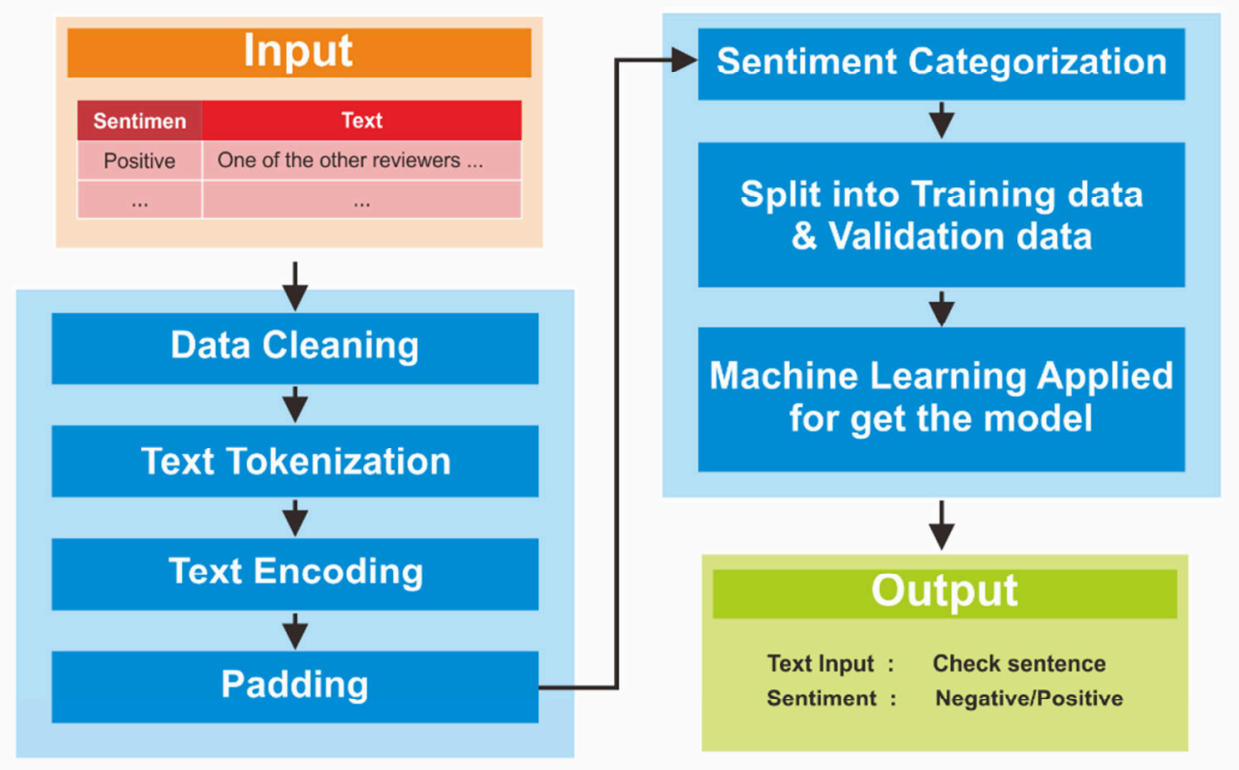

FIGURE 2. Machine learning approah for sentiment analysis 


\section{Convolutional Neural Networks}

Convolutional neural networks architecture consists of three main layers, namely the input layer, feature extraction layer, and classification layer [6]. This architecture is shown by Fig. 3. For the text classification, the Embedding process is performed on the input layer. Word embedding is procces wich represent words as dense vectors [26]. This method aims to identify the closeness between words through vector representation. The word embedding process is exemplified in Fig. 4.

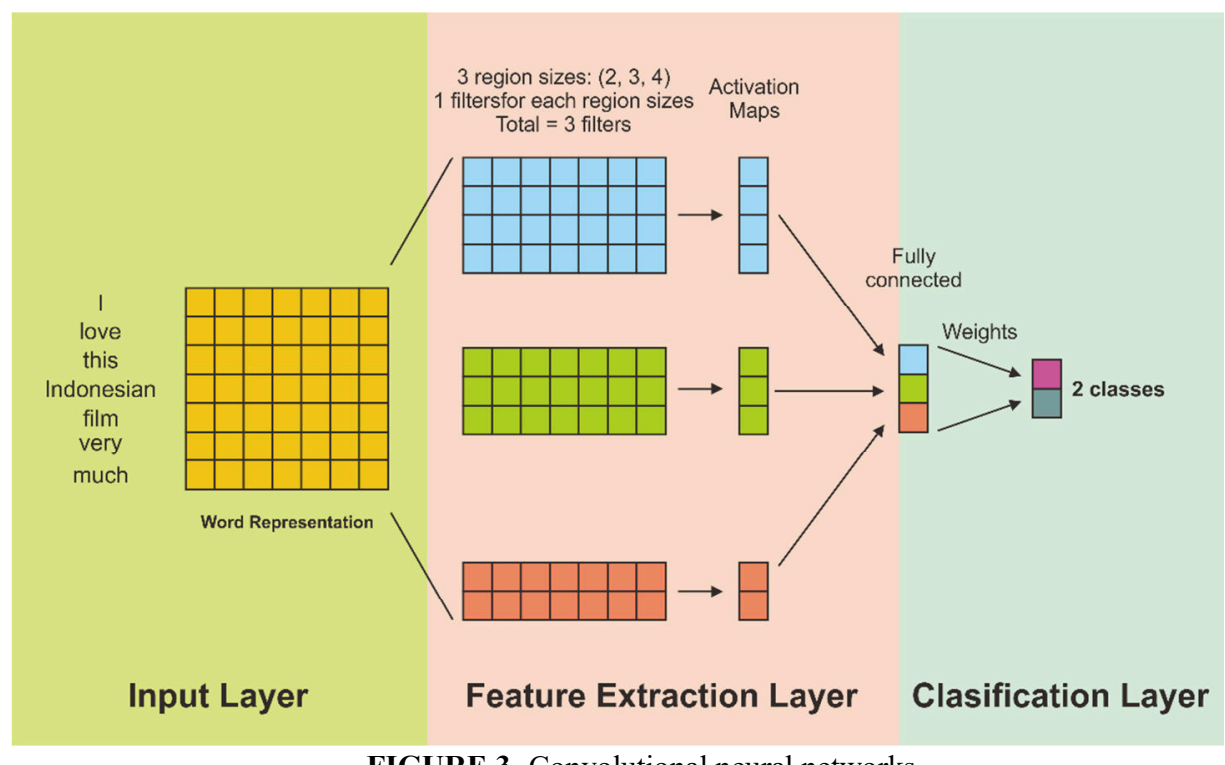

FIGURE 3. Convolutional neural networks

In the next layer, the convolution process is carried out. The term convolution refers to the mathematical combination of two functions to produce a third function [27]. It combines two sets of information. Convolution aims to extract features. The important features will only be considered in the neural network model.

\section{Word Encoding + Embedding}

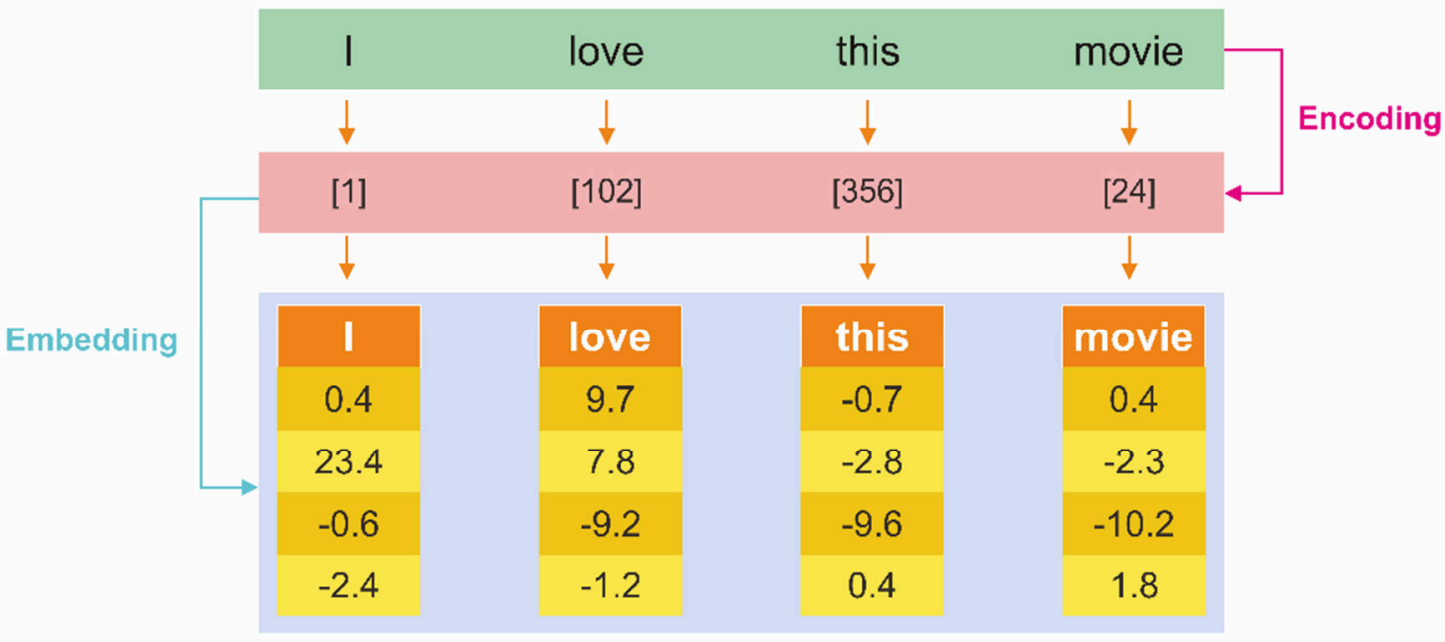

FIGURE 4. Word embedding 
We avoid overfiting by applying dropout process. Dropout is a technique where randomly selected neurons are ignored during training [28]. This means that their contribution to the activation of downstream neurons is temporally removed on the forward pass and any weight updates are not applied to the neurons on the backward pass. Dropout architecture is shown by Fig. 5.

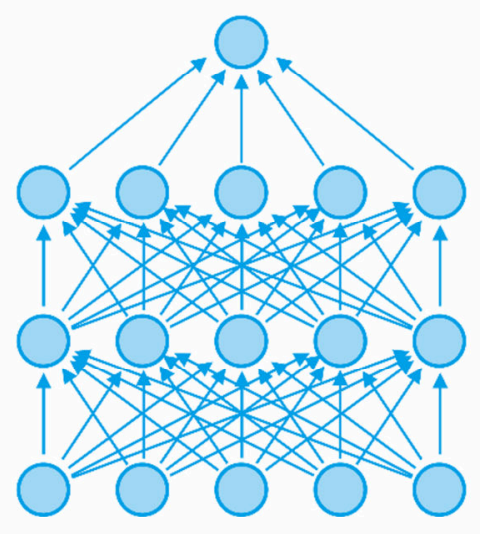

Standar Neural Networks

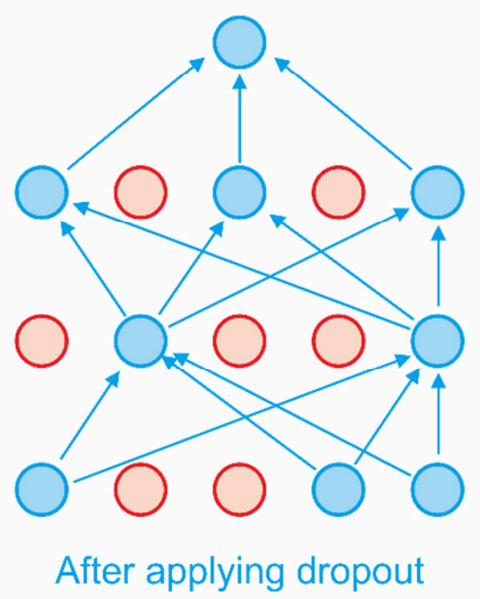

FIGURE 5. Dropout

. Maxpooling process is carried out to reduce the computational burden of this algorithm. The tip of this modeling is the classification layer to produce the desired output. For each classification, the appropriate activation function is used. For binary classification, the sigmoid activation function is used. As for the multi-class classification, the softmax activation function is used. Activation function at the other layer, in this model the relay function is used. Then the compiled model is compiled and trained to produce the desired model of convolutional neural networks. The result of this model is the probability value of each classification class. The class with the highest probability value, is considered the class of the object in question. Writing code in TensorFlow 2.0 for convolutional neural networks can be seen in Fig. 6.

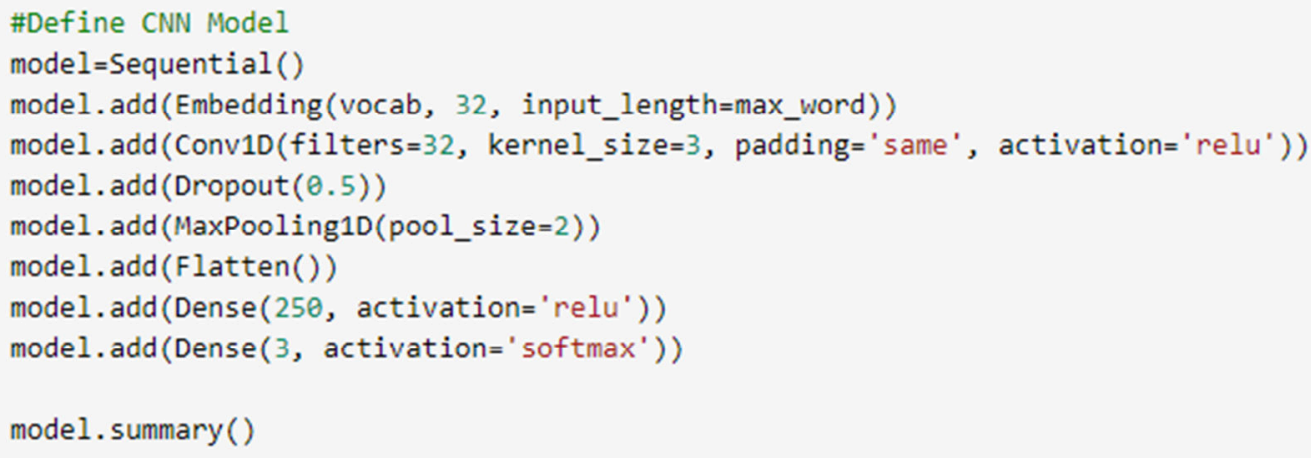

FIGURE 6. Convolutional neural networks model in Tensor Flow 2.0

\section{Fuzzy Logic}

The fuzzy logic model is based on the fuzzy set theory proposed by Zadeh [13]. Fuzzy set is a set consisting of values between 0 and 1 that describe the degree of membership of an object. If the logic of binary logic has a value 
of "Yes" and "No", then values close to 0 mean that they tend to be in the "No" decision and vice versa. In the case of sentiment analysis, two things that are binary are "positive" and "negative" sentiments. In this study, 0 is associated with "negative" sentiment and 1 is associated with "positive" sentiment. So that the values between them are the degree of sentiment of a word or sentence. The "neutral" sentiment in a sentence will be associated with the number 0.5 .

\section{Euclidean Distance}

Euclid's distance between two vectors $\vec{p}=\left(p_{1}, p_{2}, \ldots, p_{n}\right)$ dan $\vec{q}=\left(q_{1}, q_{2}, \ldots, q_{n}\right)$ in Euclidean space is defined as follows [29]:

$$
\begin{aligned}
d(\vec{p}, \vec{q}) & =d(\vec{q}, \vec{p}) \\
& =\sqrt{\left(q_{1}-p_{1}\right)^{2}+\left(q_{2}-p_{2}\right)^{2}+\cdots+\left(q_{n}-p_{n}\right)^{2}} \\
& =\sqrt{\sum_{i=1}^{n}\left(q_{i}-p_{i}\right)^{2}}
\end{aligned}
$$

\section{Datasets}

In this study a different dataset was used. The first dataset is an Indonesian text dataset taken from Twitter tweet. This tweet dataset has undergone a process of data cleaning and labeling (negative, neutral, and positive). It's the general dataset for Indonesian Sentiment Analysis [30].The second dataset is the Twitter tweet dataset for American Airlines flight services. This dataset is still in the form of a raw dataset, so it requires a data cleaning process to apply the sentiment analysis process. The final dataset is the film review dataset. This dataset is known as the IMDB Film Review dataset. Detailed information about the dataset used in this study is presented in Table 1.

TABLE 1. Sentiment Analysis Dataset.

\begin{tabular}{lccc}
\hline \multicolumn{1}{c}{ Dataset } & Sentiment Class & Records & CSV File Size \\
\hline Indonesian Sentiment Twitter & 3 & 10.806 & $962 \mathrm{~KB}$ \\
Airline Sentiment Twitter & 3 & 14.640 & $3.073 \mathrm{~KB}$ \\
IMDB Film Review & 2 & 50.000 & $64.661 \mathrm{~KB}$ \\
\hline
\end{tabular}

\section{Proposed Method}

This study seeks to produce a sentiment analysis method in the form of sentiment degree in a sentence. The model used is a convolutional neural network model. Dataset that is still raw needs to be cleaned first. Then the data preparation process is carried out with the process of tokenization, encoding and padding. Then the data is divided into training data and test data. Convolutional neural network model is applied to the data. The results of the model are the probability values of a sentence having a negative, neutral or positive sentiment class. Then the results are modified into fuzzy set. The steps of the method we propose are presented in Fig. 7.

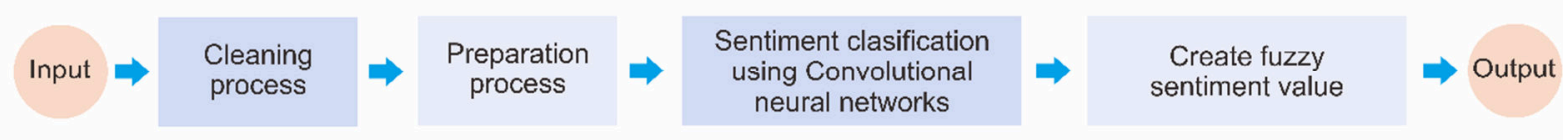

FIGURE 7. Fuzzy sentimen analysis using convolutional neural networks

Modification of the model output results to the fuzzy set is shown in Fig. 8(a) for the binner class and Fig. 8(b) for multi classes. The output of this method is the sentiment value vector of sentences in the dataset. This vector is calculated equally (This vector is calculated the similarities) with the euclid distance concept with the dataset 
sentiment label vector. The distance between the standard fuzzy sentiment vector and the standard convolutional neural network sentiment vector are compared. The smaller distance result means that the vector approaches the dataset sentiment label vector. This means that the sentiment value is better, or closer to the actual results.

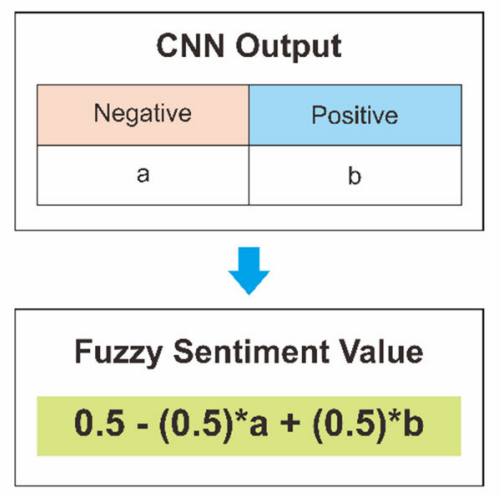

(a)

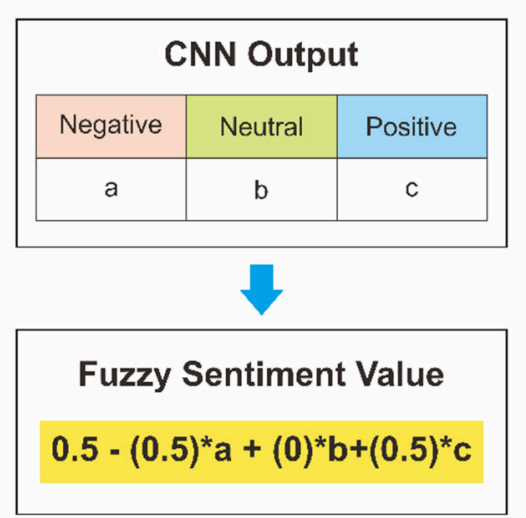

(b)

FIGURE 8. Create fuzzy sentiment value

\section{RESULT AND DISCUSSION}

Sentiment analysis model using the convolutional neural networks model is presented in Table 2. In this table, it is known that the dataset with the fewest lines of text has the smallest validation accuracy. This applies vice versa. It's indicates that by multiplying the dataset for the training process, a better model will be generated. We try to develop model with more than $90 \%$ accuracy training model, but at the same times avoid the overfitting. So, we get the result show in Table 2 . Although there is a relatively small validation accuracy value of $58.05 \%$ on Indonesian Sentiment Twitter dataset, the training accuracy value is still above $90 \%$. We used same model to all dataset, so if we want to improve that result we can apply another convolutional neural networks layer structure.

TABLE 2. Convolutional neural networks model accuracy.

\begin{tabular}{lccc}
\hline \multicolumn{1}{c}{ Dataset } & $\begin{array}{c}\text { Training } \\
\text { Accuracy }\end{array}$ & $\begin{array}{c}\text { Validation } \\
\text { Accuracy }\end{array}$ & Epoch Number \\
\hline Indonesian Sentiment Twitter & $98.44 \%$ & $53.44 \%$ & 100 \\
Airline Sentiment Twitter & $99.56 \%$ & $76.40 \%$ & 100 \\
IMDB Film Review & $99.99 \%$ & $86.88 \%$ & 40 \\
\hline
\end{tabular}

The results of Table 2 are obtained from the convolutional neural networks model with the specifications shown in Fig. 9. These results confirm that this model has high accuracy [6-8]. The lowest validation accuracy results on the dataset with minimal records confirm statements by A. Sadia, F. Khan, and F. Bashir [5]. This high validation accuracy value is generated with relatively low values of kernel and filter hyperparameter in line with the results of research [9]. The accuracy results of Twitter Dataset Airline Sentiment validation are lower than the IMDB Dilm Review dataset, the hyperparameter value of embedding dimension on Twitter Airline Sentiment dataset is designed to be larger than the last dataset ie 256 (the other dataset is only 32). Nevertheless this result is the best result for the dataset. This result has similarities with the statement state that more complicated models actually produce worse accuracy values [10]. 


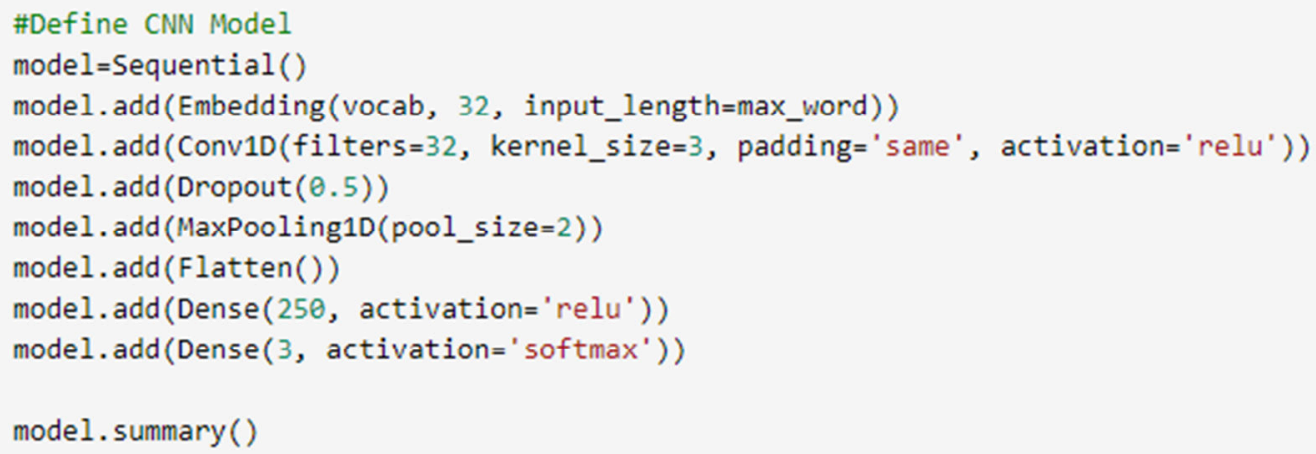

FIGURE 9. Layer configuration on convolutional neural networks model

Sentiment of a sentence is displayed in fuzzy form. The results are compared with the results of sentiment analysis with standard convolutional neural networks. The value of the sentiment degree produced is always close to either the prediction with the standard sentiment analysis or the actual sentiment label. This result is shown in Table 3 .

TABLE 3. Fuzzy sentiment analysis

\begin{tabular}{|c|c|c|c|}
\hline \multicolumn{4}{|c|}{ Indonesian Sentimen Twitter Dataset } \\
\hline Text & Sentiment Label & $\begin{array}{c}\text { Sentiment } \\
\text { Prediction using } \\
\text { CNN }\end{array}$ & $\begin{array}{l}\text { Fuzzy Sentiment } \\
\text { Prediction using } \\
\text { CNN }\end{array}$ \\
\hline $\begin{array}{l}\text { lagu bosan apa yang aku save ni } \\
\text { huhuhuhuhuhuhuhuhuhuuuuuuuuuuuuu } \\
\mathrm{u}\end{array}$ & Negatif [0] & {$[0]$} & 0.0000 \\
\hline $\begin{array}{l}\text { kita lanjutkan saja diam ini hingga kau } \\
\text { dan aku mengerti tidak semua }\end{array}$ & Negatif $[0]$ & {$[0.5]$} & 0.4986 \\
\hline $\begin{array}{l}\text { kebersamaan harus melibatkan hati } \\
\text { makasih loh ntar kita bagi hasil aku } 999 \\
\text { sisanya buat kamu }\end{array}$ & Positif [1] & [1] & 0.9994 \\
\hline $\begin{array}{l}\text { aku tak faham betul jenis orang } \\
\text { malaysia yang tak reti nak sangkut balik } \\
\text { hose tu aku geli bodoh nak pegang }\end{array}$ & Positif [1] & [1] & 0.9999 \\
\hline \multicolumn{4}{|c|}{ Airline Sentiment Twitter } \\
\hline what dhepburn said. & Neutral [0.5] & {$[0.5]$} & 0.4999 \\
\hline $\begin{array}{l}\text { plus you've added commercials to the } \\
\text { experience tacky. }\end{array}$ & Positif [1] & {$[1]$} & 0.9999 \\
\hline $\begin{array}{l}\text { i didn't today... Must mean I need to } \\
\text { take another trip! }\end{array}$ & Neutral $[0.5]$ & {$[0.5]$} & 0.4999 \\
\hline $\begin{array}{l}\text { it's really aggressive to blast obnoxious } \\
\text { "entertainment" in your guests' faces } \\
\text { \&amp; they have little recourse }\end{array}$ & Negatif [0] & [0] & 0.0000 \\
\hline \multicolumn{4}{|c|}{ IMDB Film Review } \\
\hline $\begin{array}{l}\text { One of the other reviewers has } \\
\text { mentioned that ... }\end{array}$ & Positif [1] & [1] & 1.0000 \\
\hline $\begin{array}{l}\text { A wonderful little production. The } \\
\text { filming tec... }\end{array}$ & Positif [1] & [1] & 1.0000 \\
\hline $\begin{array}{l}\text { I thought this was a wonderful way to } \\
\text { spend ti.. }\end{array}$ & Positif [1] & [1] & 0.9999 \\
\hline $\begin{array}{l}\text { Basically there's a family where a little } \\
\text { boy ... }\end{array}$ & Negatif [0] & [0] & 0.0035 \\
\hline
\end{tabular}


Fuzzy sentiment value always approaches the real sentiment label. In this study only focuses on datasets with binary classes and three classes. In contrast to the results of research by [21], which used 6 classes. In this study, the output of the convolutional neural network model as in [20] was modified to be a fuzzy sentiment value. In other studies, the concept of fuzzy logic is used to classify text sentiment classes and successfully outperform other classification methods [23]. But the results of the method are not presented in the form of degrees of sentiment.

To show that fuzzy sentiments represent sentiments of a sentence more smoothly then it is tested by the proximity test of two vectors using euclid distance. The results of fuzzy sentiment vectors with standard convolutional neural networks sentiments are presented in Fig. 10.

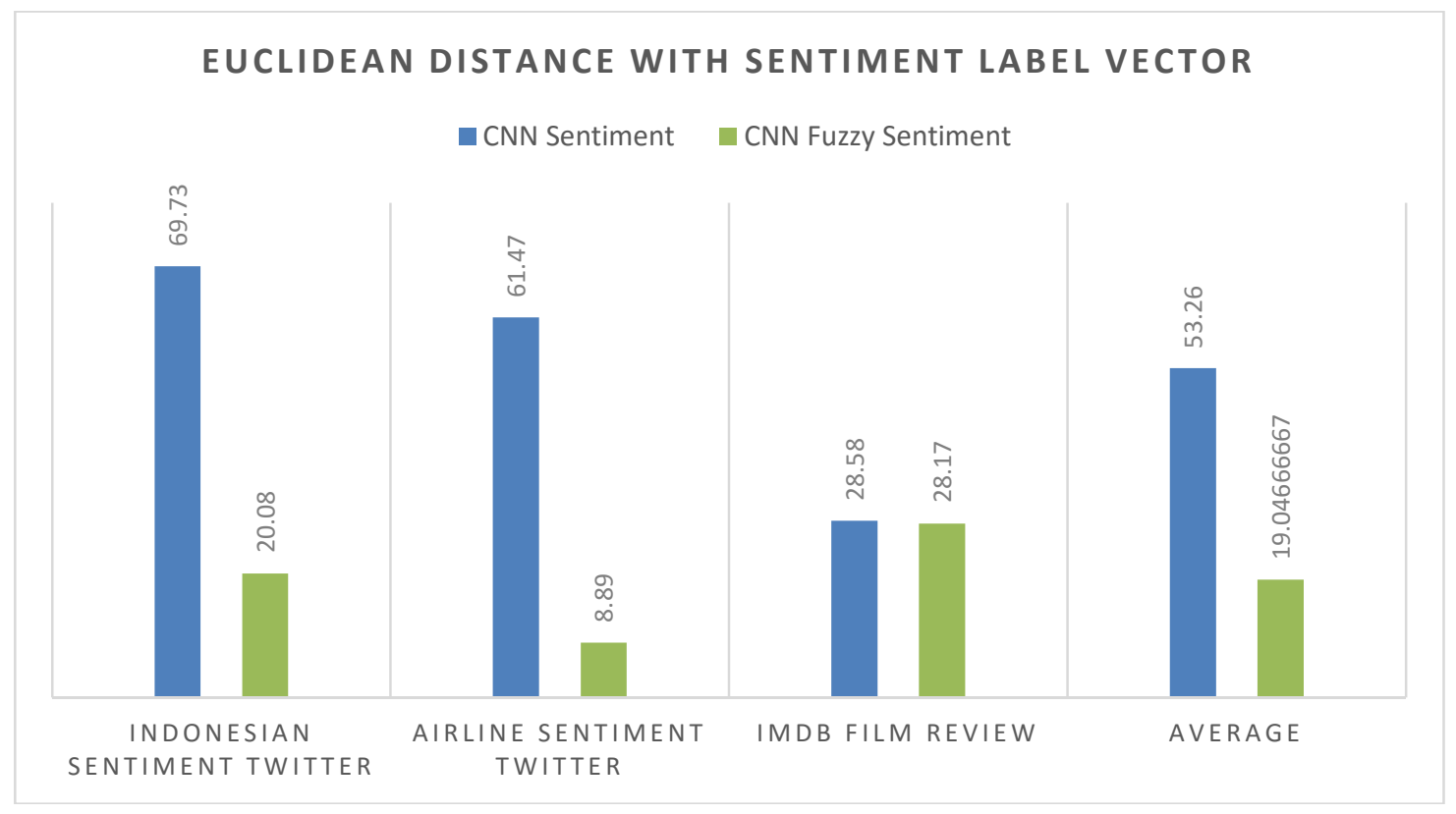

FIGURE 10. Euclidean Distance with Sentiment Label Vector

In the dataset with the three sentiment classes the method we propose produces a much smaller distance. That means that the results of our method are closer to the sentiment label vector. Although the dataset with two classes is not able to reduce the magnitude of the euclid distance, the results are relatively similar. This means that the results of our method are not much different from the standard methods in quality, but produce more relevant output. That was like our initial min goal.

\section{CONCLUSION}

The fuzzy convolutional neural networks can give a sentiment value in fuzzy form. Sentiment in fuzzy form bears a better resemblance to the original class label compared to convolutional neural networks sentiment values. The application of the fuzzy concept in the classification process until the output results are still in the form of a fuzzy set is recommended for further research. That was done to obtain the results of a more representative sentiment analysis..

\section{ACKNOWLEDGMENTS}

Thank you to Ahmad Dahlan University for its support in funding the research of the mathematics study program in 2020. Thank you also said to reviewers who have provided input and comments so that this article becomes more qualified. 


\section{REFERENCES}

1. M. R. Yaakub, M. I. . A. Latifi, and L. S. Zaabaar, "A Review on Sentiment Analysis Techniques and Applications," in Joint Conference on Green Engineering Technology \& Applied Computing2019, IOP Conference Series: Materials Science and Engineering, 551, 012070, (2019).

2. A. Alsaeedi and M. Z. Khan, "A Study on Sentiment Analysis Technique of Twitter Data," International Journal of Advance Computer Science and Applications 10, 361-374 (2019).

3. M. D. Devika, C. Sunitha, and A. Ganesh, "Sentiment Analysis: A Comparative Study On Different Approaches," in Fourth International Conference on Recent Trends in Computer Science \& Engineering, Procedia Computer Science, 87, 44-49, (2016).

4. M. Taboada, J. Brooke, M. Tofiloski, K. Voll, and M. Stede, "Lexicon-based methods for sentiment analysis," inComputational linguistics, 37(2), (Association for Computational Linguistics, 2011), pp. 267-307.

5. A. Sadia, F. Khan, and F. Bashir, "An Overview of Lexicon-Based Approach For Sentiment Analysis," in International Electrical Engineering Conference(IEEC) $3^{\text {rd }}$. (IEP Centre, Karachi, Pakistan, 2018).

6. M. A. Nasichuddin, T. B. Adji, and Widyawan, "Performance Improvement Using CNN Sentiment Analysis," International Journal of Innovative Technology and Exploring Engineering 2, No. 1, (2018).

7. Y. Zhang and B. Wallace, "A Sensitivity Analysis of (and Practitioners'Guide to) Convolutional Neural Networks for Sentence Classification,”arXiv:1510.03820 [cs.CL], (2015).

8. A. Hassan and A. Mahmood, "Deep Learning Approach fo Sentiment Analysis of Short Texts," in $3^{\text {rd }}$ International Conference Computation Analysis Security Trends, CAST, (2017), pp. 705-710.

9. K. G. Pasi and S. R. Naik, "Effect of Parameter Variations on Accuracyof Convolutional Neural Network," in International Conference Computation Analysis Security Trends, CAST, 2017, pp. 398-403.

10. X. Ouyang, P. Zhou, C. H. Li, and L. Liu, "Sentiment Analysis UsingConvolutional Neural Network," in IEEE Int. Conf. Comput. Inf.Technol. Ubiquitous Comput. Commun. Dependable, Auton. Secur.Comput. Pervasive Intell. Comput., (2015), pp. 2359-2364.

11. J.F Sánchez-Rada andC. A. Iglesias, "Social context in sentiment analysis: Formal definition, overview of current trends and framework for comparison," Information Fusion 52,344-356, (2019).

12. Q. Rajput, S. Haider, andS. Ghani, "Lexicon-based sentiment analysis of teachers' evaluation,"in Applied Computational Intelligence and Soft Computing, edited by Francesco Carlo Morabito, (Hindawi Publishing Corporation, 2016).

13. V. Nov'ak,"A New Proof of Completeness of Fuzzy Logic andSome Conclusions for Approximate Reasoning," Proc. Int. ConferenceFUZZ-IEEE/IFES'95, 1461-1468, (1995).

14. V. Nov'ak, "Ultraproduct Theorem and Recursive properties ofFuzzy Logic," Non-Classical Logics and their Applications to Fuzzy Subsets, 341-370, (1995).

15. K. K. Uma andD. K. Meenakshisundaram, " A Novel Optimizer Technique for Sentiment Polarity in Social Web Environment," International Journal of Applied Engineering Research14, (2019).

16. P. Bedi andP. Khurana P, (2020) "Sentiment Analysis Using Fuzzy-Deep Learning,"Proceedings of ICETIT 2019605, (2020).

17. Nguyen, Tuan-Linh, Swathi Kavuri, and Minho Lee. "A fuzzy convolutional neural network for text sentiment analysis,"Journal of Intelligent \& Fuzzy Systems 35, 6025-6034, (2018).

18. Howells, Karen, and Ahmet Ertugan. "Applying fuzzy logic for sentiment analysis of social media network data in marketing." Procedia computer science 120, 664-670, (2017).

19. Wang, Xinzhi, Hui Zhang, and Zheng Xu. "Public sentiments analysis based on fuzzy logic for text," International Journal of Software Engineering and Knowledge Engineering 26, 1341-1360, (2016).

20. Jefferson, Chris, Han Liu, and Mihaela Cocea. "Fuzzy approach for sentiment analysis." 2017 IEEE international conference on fuzzy systems (FUZZ-IEEE). IEEE, 2017.

21. M. Haque, "Sentiment analysis by using fuzzy logic," arXiv preprint arXiv:1403.3185 (2014).

22. R. Rajnish, "Fuzzy aspects in sentiment analysis and opinion mining," International Journal of Innovative Research in Science, Engineering and Technology 7, 7750-7755, (2016).

23. Asghar,et al, "Fuzzy-Based Sentiment Analysis System for Analyzing Student Feedback and Satisfaction" (2019).

24. Martín Abadi,et al, TensorFlow: Large-scale machine learning on heterogeneous systems, (2015), Software available from tensorflow.org. 
25. E. Boiy andM. F. Moens, "A machine learning approach to sentiment analysis in multilingual Web texts". Information retrieval12, 526-558, (2009), https://doi.org/10.1007/s10791-008-9070-z.

26. O. Levy andY. Goldberg,"Neural word embedding as implicit matrix factorization," Advances in neural information processing systems, 2177-2185, (2014).

27. A. Behl, A. Bhatia, and A. Puri, "Convolution and Applications of Convolution," International Journal of Innovative Research in Teclmology1, (2014).

28. Y. Gal, and Z. Ghahramani, "Dropout As a Bayesian Approximation: Representing Model Uncertainty in Deep Learning," in Proceedings of the 33rd International Conference on International Conference on Machine Learning (ICML-2016), (2016).

29. G. Strang, et al,"Introduction to linear algebra", Vol. 3,(Wellesley, MA: Wellesley-Cambridge Press, 1993).

30. R. Ferdiana , F. Jatmiko, D.D. Purwanti, A.S.T. Ayu, and F. Dicka, "Dataset Indonesia untuk Analisis Sentimen", Jurnal Nasional Teknik Elektro dan Teknologi Informasi (JNTETI), 8, 334-339, (2019). 